\title{
Lifangitis esclerosante no venérea del pene. Presentación de 2 casos y revisión de la literatura
}

\section{Non-venereal sclerosing lymphangitis of the penis. Cases presentation and literature review}

\author{
Wilmer Roberto Rivero Rodríguez, MD $^{1}$ Juliana Estefanía Pradilla Valbuena, MD² \\ ${ }^{1}$ Urólogo, Andrólogo, Jefe de Urología, Fundación Cardiovascular de \\ Colombia, Floridablanca, Santander, Colombia \\ 2 Universidad Autónoma de Bucaramanga, Bucaramanga, Colombia \\ Address for correspondence Juliana Estefanía Pradilla Valbuena, MD, \\ Universidad Autónoma de Bucaramanga, Bucaramanga, Colombia \\ (e-mail: julianita26@hotmail.com).
}

Urol Colomb 2018;27:287-289.

\section{Resumen \\ Palabras Clave \\ - linfangitis esclerosante \\ - enfermedad de Mondor del pene \\ - tromboflebitis}

La linfangitis esclerosante no venérea del pene es una afección benigna, caracterizada por un cordón indurado e indoloro, en la proximidad del surco balanoprepucial que puede o no estar acompañado de dolor durante las relaciones sexuales. Afecta a hombres de diferentes edades, siendo más probable en su periodo de mayor actividad sexual, que ha sido relacionada en el marco de otras enfermedades siendo su principal diagnóstico diferencial la enfermedad de Mondor del pene, en la cual se evidencia una trombosis de la vena dorsal superficial del pene. Debido a que su curso es autolimitado en la mayoría de los pacientes, existe un subregistro de los casos, además de generar controversia en su abordaje terapéutico. Presentamos 2 casos de pacientes adultos jóvenes que ingresaron a nuestra institución, y fueron diagnosticados con esta enfermedad, en el primer semestre de 2016.

Non-venereal sclerosing lymphangitis of the penis is a benign condition, characterised by a painless indurated cord-like thickening located in the proximity of the balanopreputial sulcus. It can be accompanied by pain during sexual intercourse. It affects men of different ages, being more frequent in periods with increased sexual activity, given its correlation with other sexually transmitted diseases. The main differential diagnosis is Mondor's disease of the penis, which is characterised by thrombosis of the superficial dorsal vein of the penis. Given its self-limited course in the majority of patients, the condition is underdiagnosed, and there is controversy regarding its diagnostic approach. Two cases are presented on young adult male patients admitted to our institution, and were diagnosed with this condition in the first semester of 2016.

\section{Introducción}

La linfangitis esclerosante no venérea del pene es una lesión benigna, en la mayoría de los casos autolimitada, que afecta a los hombres en su periodo de mayor actividad sexual, entre los $20 \mathrm{y}$ 45 años de edad aproximadamente, aunque puede presentarse fuera de este rango de edad..$^{1-3}$ A pesar de que se han reportado casos desde hace más de 60 años, no se ha esclarecido del todo su fisiopatología, siendo la teoría más aceptada la relacionada con traumatismos locales repetidos por relaciones sexuales vigorosas $\mathrm{y} / \mathrm{o}$ masturbación con obstrucción linfática superficial secundaria. ${ }^{1-3}$ Clínicamente se caracteriza por un received January 14, 2017 accepted April 16, 2017 published online January 10, 2018
DOI https://doi.org/ 10.1016/j.uroco.2017.04.006 ISSN 0120-789X. eISSN 2027-0119.
Copyright (c) 2018, Sociedad Colombiana License terms de Urología. Publicado por Thieme Revinter Publicações Ltda., Rio de Janeiro, Brazil. Todos los derechos reservados. 
cordón indurado, de consistencia cartilaginosa, a nivel subcoronal, móvil e indoloro en la mayoría de los casos. ${ }^{1-3}$ Por su carácter autorresolutivo sigue siendo difícil tener estadísticas actualizadas sobre su prevalencia y consenso con respecto al manejo. Presentamos el caso de unos pacientes valorados y tratados en consulta externa de urología.

\section{Casos clínicos}

En nuestra consulta nos encontramos a 2 pacientes adultos jóvenes de 20 y 42 años, previamente sanos, quienes comentan cuadro clínico de aproximadamente 2 meses de evolución caracterizado por sensación de masa a nivel distal del pene, el segundo de ellos con dolor local de intensidad leve, que se incrementa durante las relaciones sexuales, imposibilitando la finalización de las mismas. Durante sus valoraciones iniciales niegan antecedentes patológicos, infección de transmisión sexual o traumas a nivel genital. Al examen físico se evidencia en ambos una lesión indurada, levemente dolorosa a la palpación, de consistencia cartilaginosa, no adherida a planos profundos, en la región subcoronal del pene, en la zona lateral izquierda (-Figs. 1-2). No adenopatías a nivel inguinal. Se solicita ecografía doppler de pene en ambos, que fueron negativas para alteraciones en cuerpos cavernosos, tejidos blandos o venas superficiales. Se diagnostica linfangitis esclerosante no venérea del pene y se inicia manejo sintomático con betamesona tópica 2 veces al día, minociclina $100 \mathrm{mg}$ vo cada 12 h por 20 días, masaje frío local, asociado a abstinencia sexual, con poca respuesta al momento del primer control, en el paciente de 42 años, por lo cual se refuerza manejo con biprofenid $100 \mathrm{mg}$ vo al día por 10 días, con resolución completa de las lesiones y el dolor a los 3 meses aproximadamente de la primera consulta.

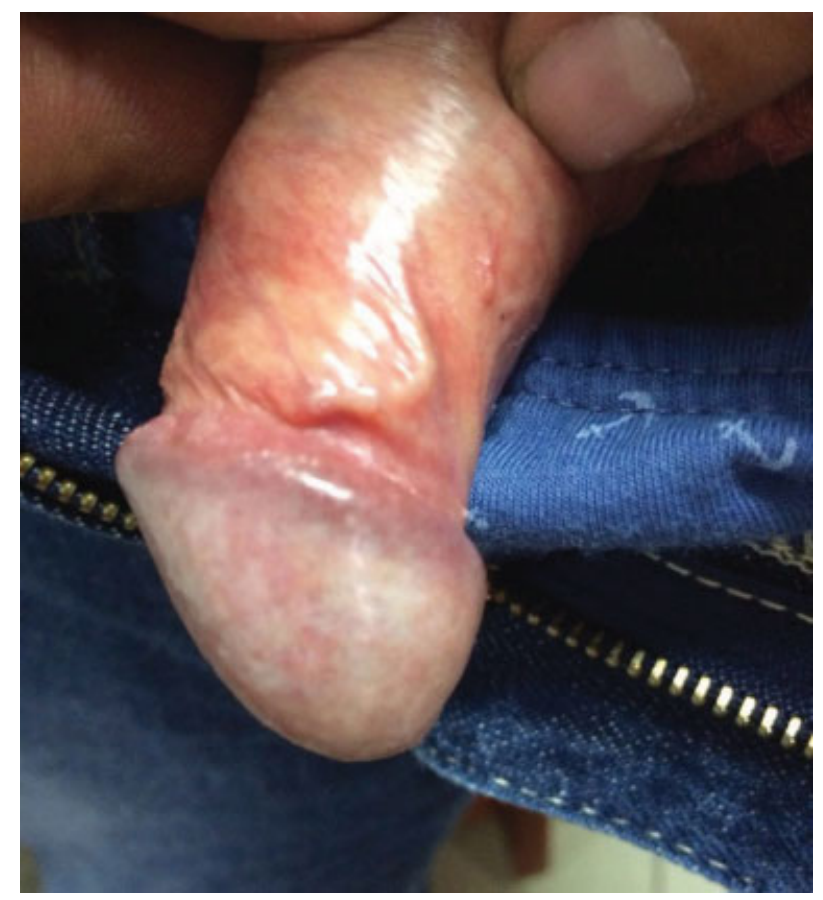

Fig. 1 Paciente de 20 años.

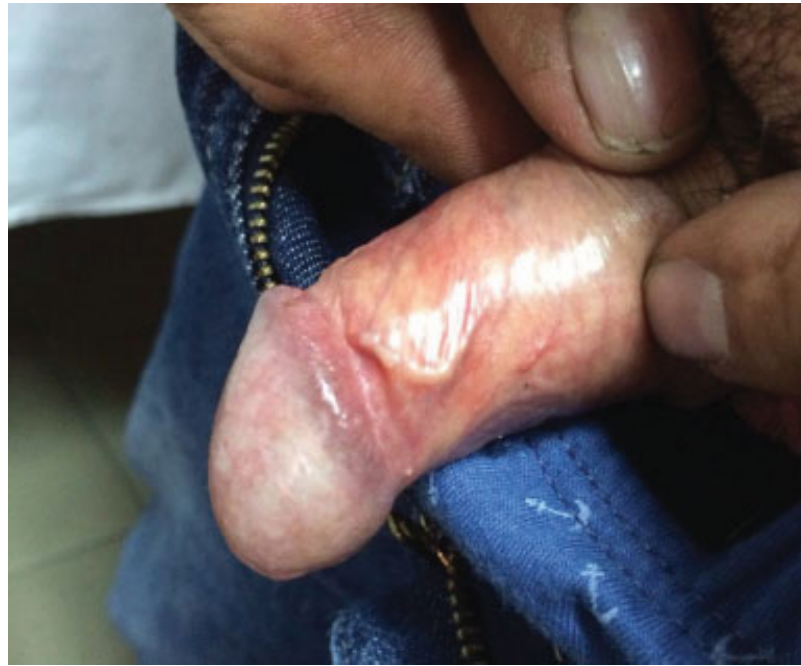

Fig. 2 Paciente de 42 años.

\section{Discusión}

La linfangitis esclerosante del pene (LEP) es una rara condición benigna que afecta a hombres jóvenes, relacionada con la etapa de mayor actividad sexual; ${ }^{1-3}$ ha tenido diferentes nombres a lo largo del tiempo como son: linfangitis indurativa circular, linfangitis transitoria benigna, linfangitis traumática $\mathrm{y}$ linfangiectasias transitorias del pene, entre otras. ${ }^{1-7}$ Fue descrita por primera vez en 1923, por Hoffman, como un episodio similar a la sífilis primaria, siendo descartada dicha asociación por él mismo, en años posteriores. ${ }^{4,5}$

Durante las últimas décadas se han encontrado similitudes de la linfangitis esclerosante no venérea del pene con la enfermedad de Mondor (EM), haciendo que hoy en día se convierta en su principal diagnóstico diferencial. Esta condición hace referencia a la tromboflebitis de venas superficiales, descrita en diferentes partes del cuerpo. ${ }^{1,2,5,7} \mathrm{Se}$ describió en 1939 por Mondor en venas torácicas, y en 1955 Braun-Falcó aplicó el término a la trombosis de la vena dorsal del pene, en contexto de un paciente con flebitis generalizada. ${ }^{5,7} \mathrm{Se}$ puede presentar en un rango más amplio de edad comparado con la LEP, pero difiere de esta entre otras cosas por los factores predisponentes, de los que cabe mencionar: tumores pélvicos, infección, hipospadias con y sin manejo, trombosis venosa profunda, drogas y fármacos endovenosos, abstinencia sexual o actividad sexual prolongada, traumatismos de repetición, entre otros.,5,6 En la LEP, se han postulado diferentes etiologías, como su asociación a procesos infecciosos y/o enfermedades de transmisión sexual. ${ }^{2-5}$ Sin embargo, la mayoría sigue estando de acuerdo en concluir que su fisiopatología está relacionada con la obstrucción de los vasos linfáticos superficiales del pene secundaria a traumatismo por actividad sexual vigorosa y/o masturbación. ${ }^{1-6}$

Con respecto a la clínica, encontramos algunas similitudes entre la LEP y la EM, lo que hace difícil su diferenciación teniendo solo en cuenta estas características, pues ambas comienzan como cordones indurados, asociados a dolor, con más frecuencia en la EM. ${ }^{7}$ La lesión no es móvil y sigue el 
trayecto de la vena dorsal superficial del pene con cambios de coloración de la piel circundante en el caso de la EM, mientras que en la linfangitis esclerosante el cordón es móvil, raramente asociado a cambios inflamatorios locales, paralelo al surco balanoprepucial. En ambos casos, las lesiones pueden ser dolorosas con la erección y/o durante el acto sexual. ${ }^{1-6}$

La ecografía doppler desempeña un papel relevante en el establecimiento de las diferencias entre estas 2 enfermedades. Mientras que en la linfangitis esclerosante se evidencian cambios inflamatorios a nivel linfático, y en algunos casos incluso puede ser reportada como normal, en la EM se evidencia un incremento en el calibre de la vena dorsal del pene, con falta de compresibilidad bajo el transductor, lesión endoluminal hipoecoica, además de cambios inflamatorios en las paredes de dicha vena con reducción o ausencia de flujo secundario. ${ }^{1,2,5}$ Varios autores infieren que es difícil diferenciar estas 2 enfermedades, e incluso se llegó a pensar que se trataba de una sola entidad, planteándose como herramienta definitiva en el diagnóstico la realización de biopsia más inmunohistoquímica; cuando esta última se lleva a cabo, se evidencia en el caso de LEP: engrosamiento de la pared de los vasos linfáticos, obstrucción parcial o total de su luz e incluso signos inflamatorios crónicos. Sin embargo, esta resulta ser una técnica dispendiosa, dolorosa y costosa, sin que conlleve beneficios adicionales en cuanto al tratamiento o pronóstico, por lo cual no es muy utilizada en la actualidad. ${ }^{1-3,5,7}$

Por todo lo anterior, la anamnesis y el examen físico, con el apoyo diagnóstico de una ecografía doppler del pene, se convierten en las principales herramientas para llegar a un diagnóstico apropiado y un mejor enfoque hacia el tratamiento.

Por último, en referencia al tratamiento de la LEP, como se ha mencionado previamente, en la mayoría de los casos la lesión desaparece con la abstinencia sexual en un periodo variable de 3 a 8 semanas. ${ }^{1-5}$ Sin embargo, cuando se quiere celeridad en la resolución se encuentran diversas estrategias entre las que están los antiinflamatorios tópicos y orales, medidas locales de frío, e incluso uso de antibióticos y resección quirúrgica de la lesión, en casos refractarios a medidas iniciales, sin que una u otra terapia haya demostrado ser superior a las otras. ${ }^{1,2,6}$ Es importante hablar con los pacientes sobre la posible recurrencia de estos episodios. ${ }^{1,3}$

\section{Conclusión}

La LEP es una rara lesión benigna de origen linfático, que afecta predominantemente a hombres jóvenes, posterior a periodos de importante actividad sexual y/o de masturbación, de curso benigno y buen pronóstico a mediano plazo, que requiere de una anamnesis y abordaje diagnóstico adecuado, para diferenciarla de enfermedades como la EM, que, si bien tienen similitudes en su clínica, presentan fisiopatología y algunos patrones de manejo diferentes.

\section{Responsabilidades éticas}

\section{Protección de personas y animales}

Los autores declaran que para esta investigación no se han realizado experimentos en seres humanos ni en animales.

\section{Confidencialidad de los datos}

Los autores declaran que han seguido los protocolos de su centro de trabajo sobre la publicación de datos de pacientes.

Derecho a la privacidad y consentimiento informado Los autores declaran que en este artículo no aparecen datos de pacientes.

Financiación

No hubo fuentes de financiación.

Conflicto de intereses

No conflicto de intereses.

\section{Bibliografía}

1 Papeš D1, Altarac S, Antabak A, Savić I. Nonvenereal sclerosing lymphangitis of the penis. Acta Dermatovenerol Croat 2015;23 (02):150-151

2 Rodríguez T. Linfangitis esclerosante del pene: Presentación de caso. Revista Habanera de Ciencias Médicas 2014;13(05): 701-707

3 Kakkanatt A, Krishnan P, Divakaran D. Sclerosing lymphangitis of penis. Literature review and report of 2 cases. Dermatology Online Journal 2014;20(7)

4 Piquero J, Piquero J, Hernández R. Linfangitis esclerosante del pene: A propósito de dos casos. Dermatología Venezolana 2005;43(3)

5 Ramos E, Portillo JA, Correas M, Gutiérrez JL, Aguilera C, Ballestero $D$, et al. Enfermedad de Mondor versus linfangitis esclerosante de pene. Arch Esp Urol 2008;61(07):837-840

6 Mateos Blanco J, Ramírez Zambrana A, Molina Suárez JL, Alarcón del Viejo C, Cabello Padial J, Mariño del Real J, et al. Linfangitis esclerosante no venérea del pene. Presentación de caso clínico. Arch Esp Urol 2007;60(03):298-300

7 Kumar B, Narang T, Radotra BD, Gupta S. Mondor's disease of penis: A forgotten disease. Sex Transm Infect 2005;81:480-482 